\title{
PRR-CERAMIC MONOCOMPOSITE AND CERAMIC COATINGS BY SOL-GEL-PYROLYSIS AND SLURRY-PYROLYSIS PROCESSING
}

\author{
BY \\ T. Sugama
}

RECEIVED

SEP $0>1999$

OSTI

Brookhaven Mational Laboratory

JANUARY 29， 1997 


\section{DISCLAIMER}

This report was prepared as an account of work sponsored by an agency of the United States Government. Neither the United States Government nor any agency thereof, nor any of their employees, make any warranty, express or implied, or assumes any legal liability or responsibility for the accuracy, completeness, or usefulness of any information, apparatus, product, or process disclosed, or represents that its use would not infringe privately owned rights. Reference herein to any specific commercial product, process, or service by trade name, trademark, manufacturer, or otherwise does not necessarily constitute or imply its endorsement, recommendation, or favoring by the United States Government or any agency thereof. The views and opinions of authors expressed herein do not necessarily state or reflect those of the United States Government or any agency thereof. 


\section{DISCLAIMER}

Portions of this document may be illegible in electronic image products. Images are produced from the best available original document. 


\section{Outline of Presentation}

1. Coating Systems

2. Starting Materials

3. Properties of Coatings 


\section{COATIhg Systens}

BINARY PHASES:

1) $\mathrm{TIO}_{2}-\mathrm{SIO}_{2}$

2) $\mathrm{ZRO}_{2}-\mathrm{SIO}_{2}$

3) $\mathrm{ZRO}_{2}-\mathrm{TrO}_{2}$

4) $\mathrm{Y}_{2} \mathrm{O}_{3}-\mathrm{ZRO}_{2}$

TERNARY PHASES:

5) $\mathrm{P}_{2} \mathrm{O}_{5}-\mathrm{TIO}_{2}-\mathrm{K}_{2} \mathrm{O}$

6) $\mathrm{LIO}_{2}-\mathrm{AL}_{2} \mathrm{O}_{3}-\mathrm{SIO}_{2}$ 


\section{COATINg Systems}

SOL-GEL

PRECURSORS
SINGLE, BINARY AND

TERNARY PYROGENIC PHASES

- Polysilazane

SILICON NITRIDE

- Polyborazinlyamine

$\longrightarrow$ BORON NITRIDE

- TI AND SI ALKOXIDE BLEND $\longrightarrow \mathrm{TIO}_{2}-\mathrm{SIO}_{2}$ SYSTEMS

- $\mathrm{ZR}_{\mathrm{R}}$ AND SI ALKOXIDE BLEND $\longrightarrow \mathrm{ZRO}_{2}-\mathrm{SIO}_{2}$ SYSTEMS

- ZR AND TI ALKOXIDE BLEND $\longrightarrow \mathrm{ZRO}_{2}-\mathrm{TIO}_{2}$ SYSTEMS

- Al AND SI ALKOXIDE BLEND $\longrightarrow \mathrm{SIO}_{2}+$ MULLITE SYSTEMS

- $Y$ AND ZR ALKOXIDE BLEND $\longrightarrow$ YITTRIA-STABILIZED ZIRCONIA

- $\mathrm{P}, \mathrm{K}$, AND TI ALKOXIDE BLEND $\rightarrow \mathrm{P}_{2} \mathrm{O}_{5}-\mathrm{TIO}_{2}-\mathrm{K}_{2} \mathrm{O}$ SYSTEMS 
STARTIMG MATERTALS

- ZIRCONIUM-N-PROPOXIDE (ZNP) 70\% IN PROPANOL $\mathrm{ZR}\left[\mathrm{O}\left(\mathrm{CH}_{2}\right)_{2} \mathrm{CH}_{3}\right]_{4}$

- Yttrium acetate (YA) 99.9\%

$\mathrm{Y}\left(\mathrm{OOCCH}_{3}\right)_{3} \cdot 4 \mathrm{H}_{2} \mathrm{O}$

- Aluminum Sec-butoxide (ASB) 95\%

$\mathrm{AL}\left(\mathrm{OC}_{4} \mathrm{H}_{9}\right)_{3}$

- Diethanolamine (DeA)

$\left(\mathrm{HOCH}_{2} \mathrm{CH}_{2}\right)_{2} \mathrm{NH}$

- ISOPROPYL ALCOHOL (IPA)

$\mathrm{CH}_{3} \mathrm{CHOHCH}_{3}$

- HYDROCHLORIC ACID

$H C L$

- WATER 


\section{Basic Formulation}

$\underline{\mathrm{ZRO}}_{2}$

ZNP DEA HCL WATER IPA

$29.4 \% \quad 44.1 \% \quad 2.9 \% \quad 8.8 \% \quad 14.7 \%$

PH 9.45

$\underline{A L}_{2} \underline{0}_{3}$

$\underline{A S B} \underline{D E A} \underline{H C L}$ WATER IPA

$32.2 \% \quad 48.4 \% 3.2 \% \quad-\quad 16.1 \%$

PH 9.09

$\underline{Y}_{2} \underline{O}_{3}$

YA DEA HCL WATER IPA

$12.6 \% 68.4 \% \quad 3.2 \% \quad-\quad 15.8 \%$

PH 9.30 
Table 1

Pane

No.

$2 r \cdot 1$

$\mathrm{Zr} \cdot 2$

AT-I

A $7 \cdot 2$

$100 \% \mathrm{Al}_{2} \mathrm{O}_{3}$

$100 \% \mathrm{Al}_{2}^{2} \mathrm{O}_{3}$

$\mathrm{YZr}-1-1 \quad 5 \% \mathrm{Y}_{2} \mathrm{O}_{3}-95 \% \mathrm{ZrO}_{2}$

$\mathrm{YZr}-1.2 \quad 5 \% \mathrm{Y}_{2} \mathrm{O}_{3}-95 \% \quad \mathrm{ZrO}_{2}$

$\mathrm{YZr}-2-1 \quad 10 \% \quad \mathrm{Y}_{2} \mathrm{O}_{3}-90 \% \quad \mathrm{ZrO}_{2}$

$\mathrm{YZr}-2.2 \quad 10 \% \mathrm{Y}_{2}^{2} \mathrm{O}_{3} \cdot 90 \% \mathrm{ZrO}_{2}$

$\begin{array}{ll}\mathrm{Y}_{2} \mathrm{O}_{3} \cdot 0 \cdot 1^{*} & 100 \% \mathrm{Y}_{2} \mathrm{O}_{3} \\ \mathrm{Y}_{2} \mathrm{O}_{3} \cdot 0-2 & 100 \% \mathrm{Y}_{2} \mathrm{O}_{3}\end{array}$

$\mathrm{Y}_{2} \mathrm{O}_{3} \cdot 1-1^{\text {t* }} \quad 100 \% \mathrm{Y}_{2} \mathrm{O}_{3}$

$\mathrm{Y}_{2}^{2} \mathrm{O}_{3}-1-2 \quad 100 \% \mathrm{Y}_{2}^{2} \mathrm{O}_{3}^{3}$

$\begin{array}{ll}Y_{2} O_{3}-2 \cdot 1^{-3} & 100 \% Y_{2} O_{3} \\ Y_{2}^{2} O_{3}-2 \cdot 2 & 100 \% \\ Y_{2} O_{3}\end{array}$
Weight of uncoated

panel. $g$

27.7967

26.5529

28.1437

26.7626

26.5121

26.5243

34.7744

26.8056

28.0463

26.9981

27.5683

26.5180

26.2032

26.8739
Total weight of coated panel, $g$

27.8036

26.5600

28.1492

26.7676

26.5305

26.5427

34.7860

26.8169

28.0587

27.0099

27.5773

26.5275

26.2072

26.8778
Weight of coating, $g$
0.0069
0.0071

0.0055

0.0055

0.0184

0.0184

0.0116

0.0113

0.0124

0.0118

0.0090

0.0095

0.0040

0.0039

* derived from 60 wt\% $\mathrm{Y}_{2} \mathrm{O}_{3}$ solution

* derived from 40 wt\% $\mathrm{Y}_{2}^{2} \mathrm{O}_{3}$ solution

*** derived form 20 wt\% $\mathrm{Y}_{2}^{2} \mathrm{O}_{3}$ solution 
SLURRY CoATIngS

LIQUID REACTANT

$-\left[-\mathrm{MPO}_{3}-\right]-\mathrm{n}$

$-\left[-\mathrm{MPO}_{3}-\right]-{ }_{n}$

$-\left[-\mathrm{MPO}_{3}-\right]-{ }_{\mathrm{n}}$

$-\left[-\mathrm{MPO}_{3}-\right]-{ }_{n}$

$-\left[-\mathrm{M}_{2} \mathrm{O} \cdot \mathrm{SrO}_{2}-\right]-{ }_{n}$

$-\left[-\mathrm{M}_{2} \mathrm{O} \cdot \mathrm{SIO}_{2}-\right]-{ }_{n}$

$-\left[-\mathrm{M}_{2} \mathrm{O} \cdot \mathrm{SrO}_{2}-\right]-{ }_{n}$

$-\left[-\mathrm{M}_{2} \mathrm{O} \cdot \mathrm{SIO}_{2}-\right]-{ }_{n}$

$\begin{array}{cc}\text { SLID REACTANT } & \begin{array}{c}\text { PYROGENIC } \\ \text { MAJOR PHASE: }\end{array} \\ \mathrm{CAO}_{\mathrm{A}} \cdot \mathrm{AL}_{2} \mathrm{O}_{3} & \rightarrow \mathrm{CAO} \cdot \mathrm{P}_{2} \mathrm{O}_{5} \\ \mathrm{CAO} \cdot \mathrm{SIO}_{2} & \rightarrow\end{array}$

$\mathrm{AL}_{2} \mathrm{O}_{3} \cdot \mathrm{SIO}_{2} \rightarrow \mathrm{M}_{2} \mathrm{O} \cdot \mathrm{AL}_{2} \mathrm{O}_{3} \cdot \mathrm{SIO}_{2}$

$\mathrm{MGO} \cdot \mathrm{AL}_{2} \mathrm{O}_{3} \rightarrow$

$\mathrm{CAO} \cdot \mathrm{AL}_{2} \mathrm{O}_{3} \rightarrow$

$\mathrm{CAO} \cdot \mathrm{SIO}_{2} \rightarrow$

$\mathrm{AL}_{2} \mathrm{O}_{3} \cdot \mathrm{SrO}_{2} \rightarrow$

$\mathrm{MGO} \cdot \mathrm{AL}_{2} \mathrm{O}_{3} \rightarrow$

$\mathrm{M}: \mathrm{NH}_{4}^{+}, \mathrm{NA}^{+}, \mathrm{K}^{+}, \mathrm{OR} \mathrm{LI}^{+}$ 


\begin{tabular}{|c|c|c|c|c|c|c|}
\hline & $\begin{array}{l}\text { Type } \\
\text { İZ } \\
\text { Cement }\end{array}$ & $\begin{array}{l}\text { Class } \\
\text { C } \\
\text { Ely Ash }\end{array}$ & $\begin{array}{l}\text { Meta- } \\
\text { Raolin }\end{array}$ & $\begin{array}{l}\text { GGBF } \\
\text { SLag }\end{array}$ & $\begin{array}{l}\text { Class } \\
F \\
\text { FIY Ash }\end{array}$ & $\begin{array}{c}\text { Silica } \\
\text { Fume }\end{array}$ \\
\hline 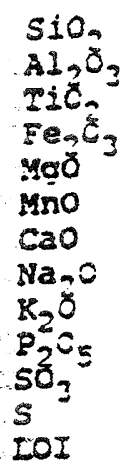 & $\begin{array}{r}20.20 \\
2.32 \\
0.21 \\
4.47 \\
3.34 \\
0.05 \\
63.90 \\
0.22 \\
0.49 \\
0.21 \\
3.00 \\
0.85\end{array}$ & $\begin{array}{l}39.50 \\
16.90 \\
6.40 \\
6.30 \\
\\
24.30 \\
1.44 \\
0.53 \\
1.99 \\
0.22\end{array}$ & $\begin{array}{r}59.91 \\
39.68 \\
0.54 \\
0.93\end{array}$ & $\begin{array}{r}35.60 \\
10.40 \\
1.26 \\
12.84 \\
37.59 \\
0.07 \\
0.33 \\
\\
0.67 \\
1.00^{\star}\end{array}$ & $\begin{array}{l}53.00 \\
18.60 \\
15.60 \\
1.26 \\
6.37 \\
0.94 \\
2.08 \\
2.02 \\
1.68\end{array}$ & $\begin{array}{l}95.86 \\
0.36 \\
0.05 \\
0.05 \\
0.25 \\
0.03 \\
0.31 \\
0.16 \\
0.68 \\
0.09 \\
0.10 \\
0.80\end{array}$ \\
\hline
\end{tabular}

GGaE - Ground granulated blast furnace

- note - gail on ignition indicates the presence of sulfur as $s$ Lather $\tan \mathrm{SO}_{2}$ 


\section{Glass Ceramic Coatings from Fly ash AS INDUSTIAL WAST}

FORMULATION: 60 WT\% FLY ASH F - 16 WT\% $-\left[-\mathrm{NAPO}_{3}-\right]-_{n}-$ 24 WT\% WATER

Dipping panels into slurry at $25^{\circ} \mathrm{C}$

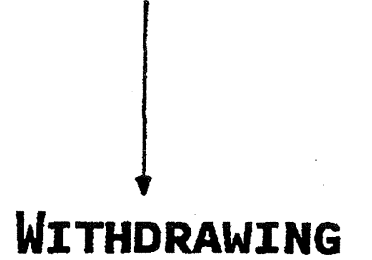

1

Preheat in aIr OVEn fOR 5 hrs at $200^{\circ} \mathrm{C}$

AnNeAling fOR 12 hRS AT $900^{\circ} \mathrm{C}$ 
Sample: FAE200

Size: $\quad 31.6010 \mathrm{mg}$

Method: TGA-900 $=15$
TGA

$-$
File: C: NiC. 042

Operator: NC

Run Date: 8-Jan-97 09: 15

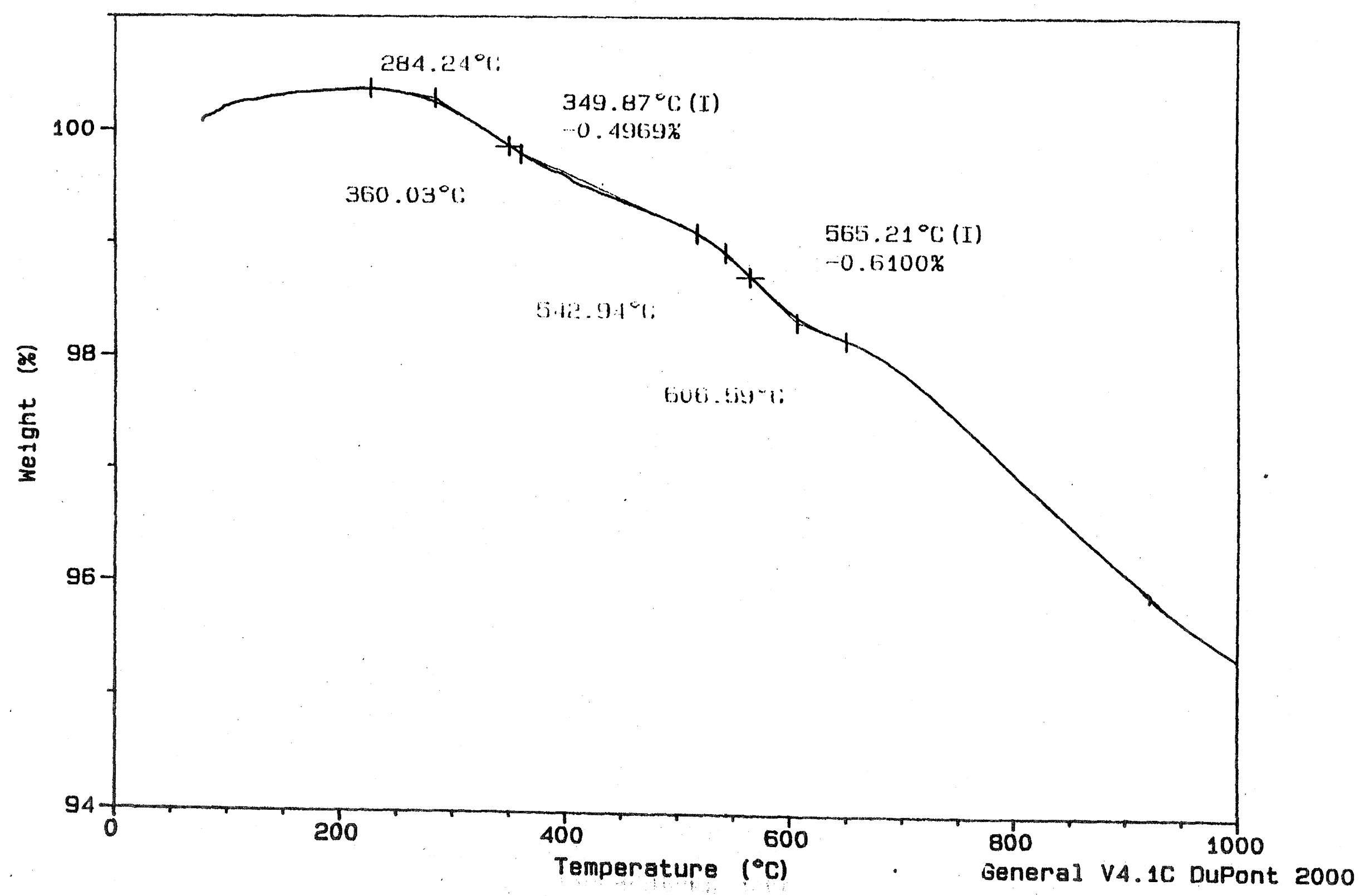




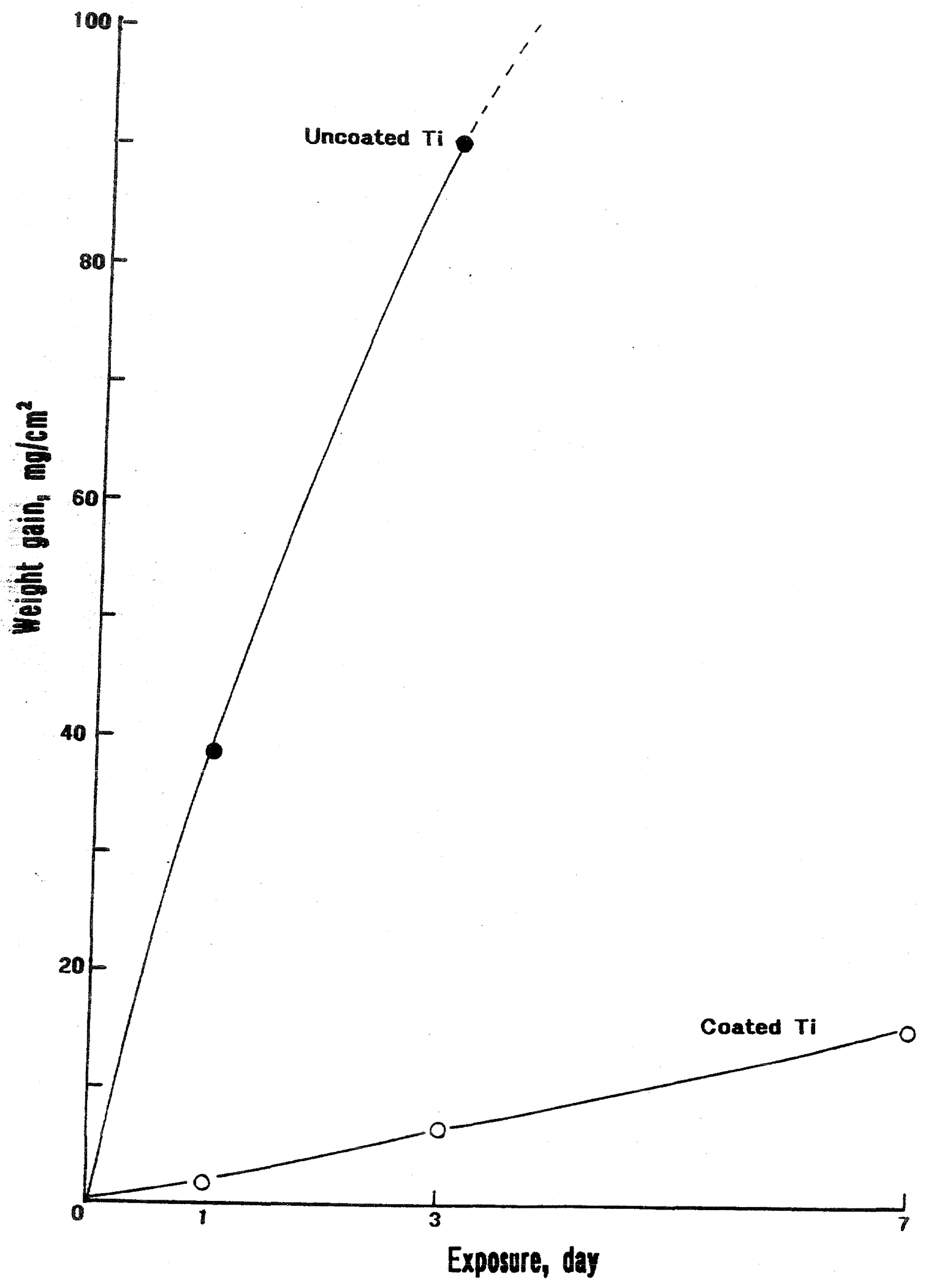




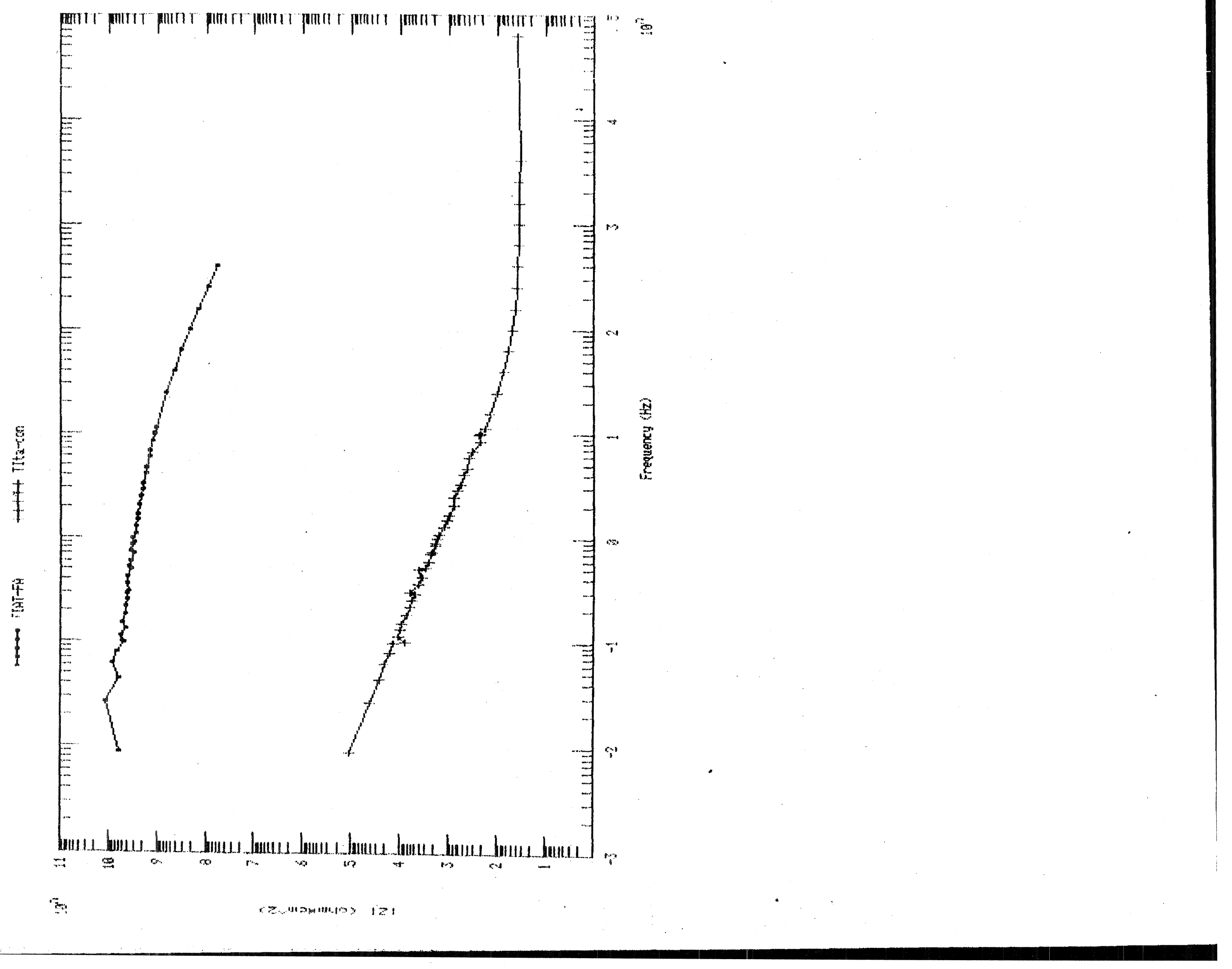


XPS Atomic Fraction of Fly Ash-Derived Coating (FA)/Ti Interfaces

\begin{tabular}{|c|c|c|c|c|c|c|c|c|c|}
\hline \multirow[b]{2}{*}{ Specimen } & \multirow[b]{2}{*}{ Failure side } & & \multicolumn{7}{|c|}{ Atomic composition, \% } \\
\hline & & $\underline{\text { Al }}$ & $\underline{\text { Si }}$ & $\underline{\mathbf{P}}$ & $\underline{\mathrm{C}}$ & Ti & $\underline{0}$ & $\underline{\mathrm{Na}}$ & $\underline{\mathrm{Fe}}$ \\
\hline $25^{\circ} \mathrm{C} \mathrm{TI}$ & - & 9.88 & 12.78 & - & 23.85 & 6.67 & 46.82 & - & - \\
\hline $900^{\circ} \mathrm{C} \mathrm{TI}$ & - & 25.51 & 4.72 & - & 7.82 & 6.92 & 55.04 & - & - \\
\hline $900^{\circ} \mathrm{C} \mathrm{FA} / \mathrm{TI}^{\circ}$ & - & 4.72 & 12.20 & 1.20 & 20.24 & 3.30 & 48.20 & 4.13 & 6.01 \\
\hline $900^{\circ} \mathrm{C} \mathrm{FA} / \mathrm{Ti}$ & FA & 16.56 & 6.12 & 12.49 & 5.71 & 6.15 & 50.79 & 2.18 & - \\
\hline $900^{\circ} \mathrm{C} \mathrm{FA} / \mathrm{TI}$ & $\mathrm{TI}$ & 10.49 & 2.72 & 9.24 & 13.51 & 12.17 & 48.37 & 3.50 & - \\
\hline
\end{tabular}




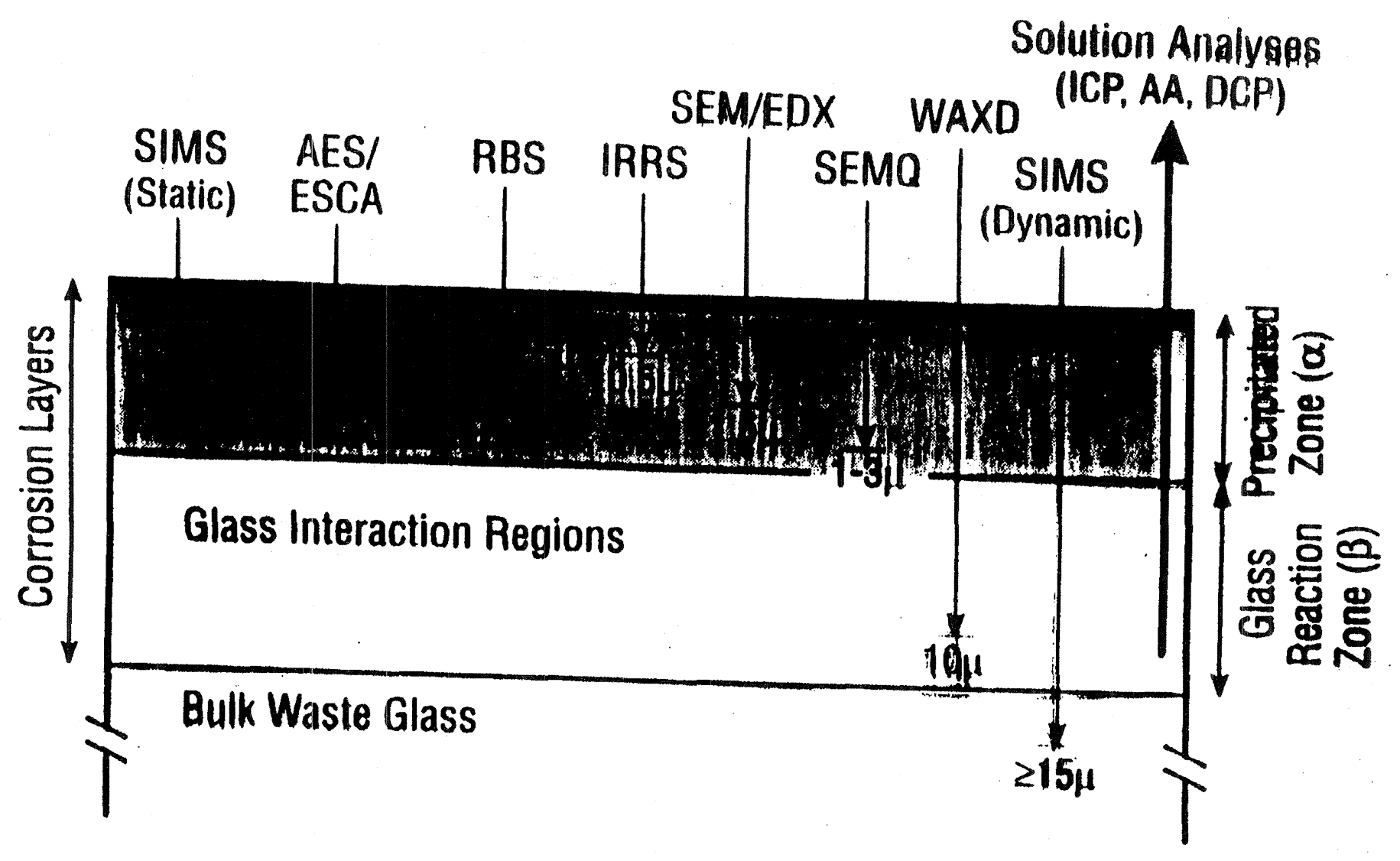




\section{TASKS}

1) SYNTHESIS AND PROPERTIES OF COLLOIDAL METALORGANIC PRECURSOR SOLUTIONS. (BNL)

2) Surface preparation of substrates (P\&W AND BNL)

3) WETTING AND SPREADING BEHAVIORS OF PRECURSORS OVIER PRE-TREATED SUBSTRATE SURFACES (BNL)

4) SCREening OF PRECURSORS (BNL)

5) FilM-FORMING PERFORMANCE OF PRECURSORS ON SUBSTRATE SURFACES AT TEMPERATURES IN THE RANGE 400 TO $1200^{\circ} \mathrm{C}$ (BNL)

6) CoRrosion, oxidATION, and WeAR REsistance tests (P\&W)

7) SCREENING OF COATING SYSTEMS, AND PATENT APPLICATIONS (BNL AND P\&W) 

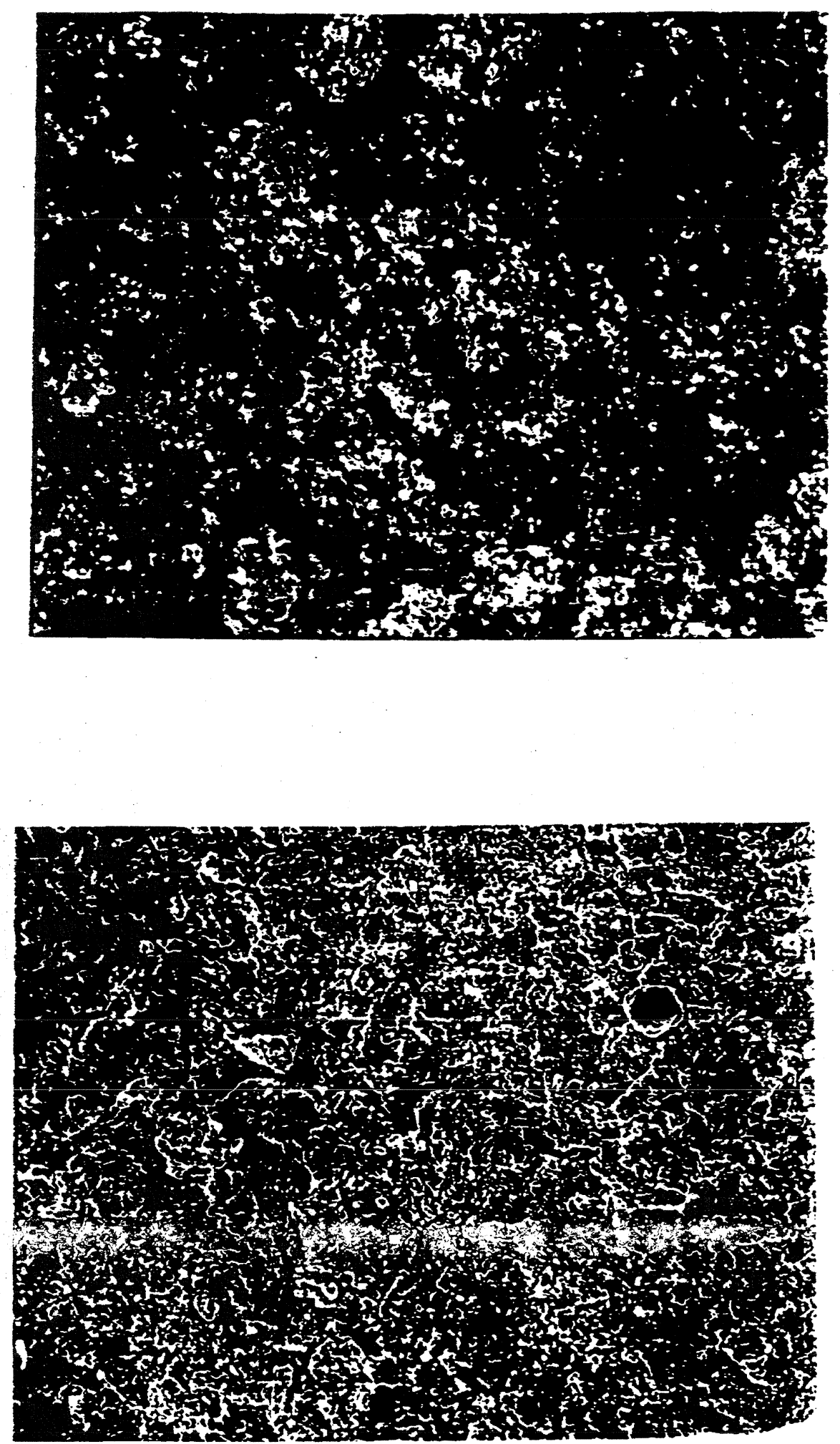


\section{Polarization Curves for $Y_{2} O_{2}$-CoAted Stainless Steels}

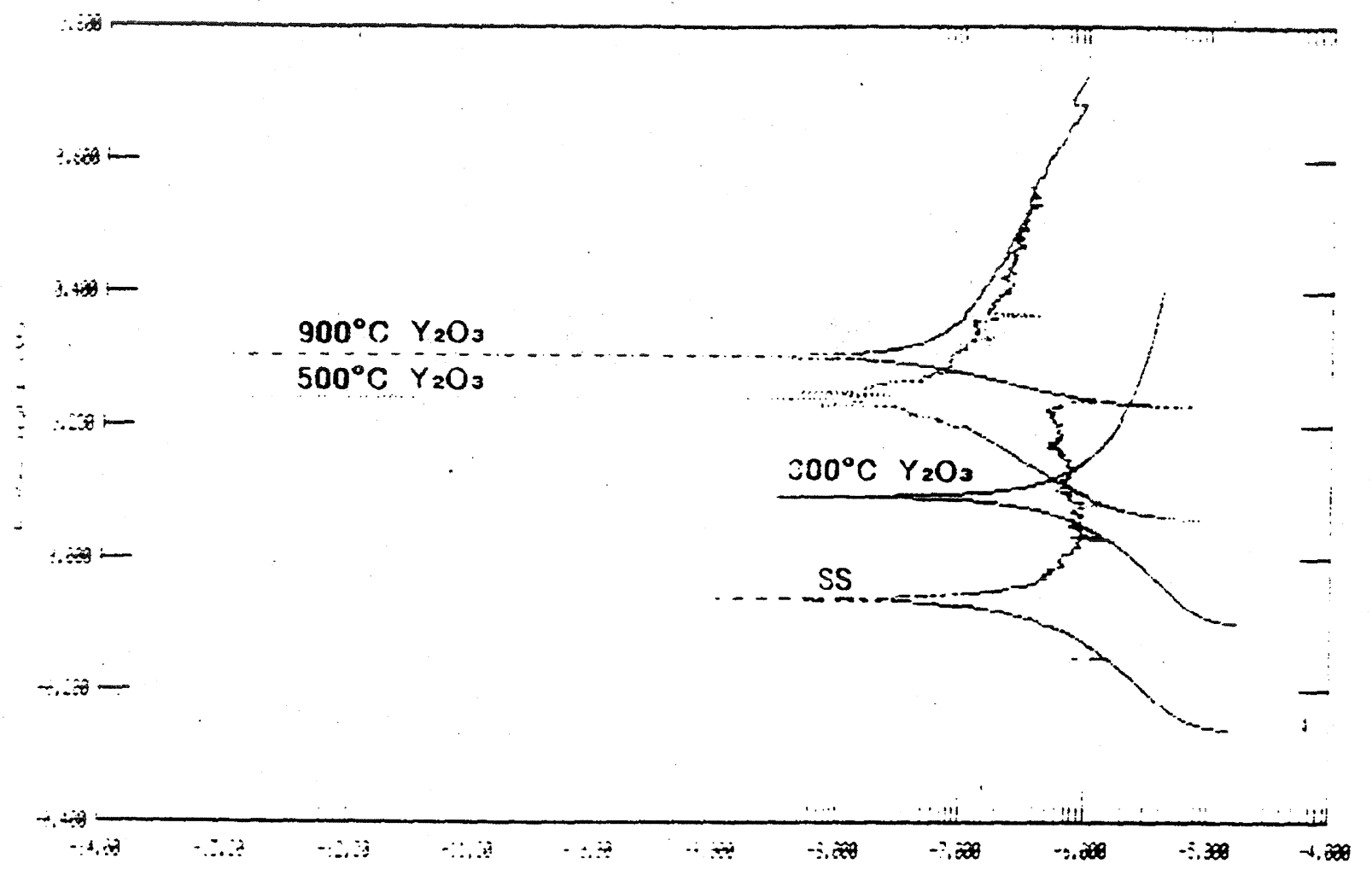

\section{TREATMENT TEMPERATURE}

COATING OF $Y_{2} \underline{0}_{3}$ PRECURSOR, ${ }^{\circ} \mathrm{C}$

STATNLESS

STEEL (SS)

$\mathrm{Y}, \mathrm{O}_{3} / \mathrm{SS}$

300

$\mathrm{Y}_{2} \mathrm{O}_{3} / \mathrm{SS}$

500

$Y, 0 . / 55$
CORROSION RATE MILLI-INCHES/YEAR
$5.17 \times 0^{-1}$

$1.80 \times 10^{-1}$

$2.17 \times 10^{-2}$

$1.08 \times 10^{-2}$ 